RIIE (2020), Año 11 (14), 82-93.

DOI: http://dx.doi.org/10.30972/riie.11144639
Resultados de la implementación del Aprendizaje Basado en Problemas en una cátedra de Ciencias Básicas en Ingeniería.

\title{
RESULTADOS DE LA IMPLEMENTACIÓN DEL APRENDIZAJE BASADO EN PROBLEMAS EN UNA CÁTEDRA DE CIENCIAS BÁSICAS EN INGENIERÍA
}

\section{RESULTS REPORT OF PROBLEM BASED LEARNING IMPLEMENTATION IN A UNIVERSITY CHAIR OF ENGINEERING BASIC SCIENCES}

\author{
Carmen Graciela Del Valle ${ }^{1}$ - Nancy Francisca Aguilar ${ }^{2}$ - Ana María Montenegro ${ }^{3}$
}

Fecha de recepción: 23-07-2020

Fecha de aceptación y versión final: 05-12-2020

\section{Resumen}

El Grupo de Investigación Educativa sobre Ingeniería (GIESIN), de la Facultad Regional Resistencia (FRRe) de la Universidad Tecnológica Nacional (UTN), Argentina, desde el año 2010 realiza investigaciones sobre educación en ingeniería. En un primer Proyecto denominado "Relación entre las competencias reales de los aspirantes y las requeridas a los ingresantes en las carreras que se dictan en la FRRe de la U.T.N." (2010-2013), se analizaron las competencias matemáticas de los estudiantes que estaban cursando primer año, mediante la aplicación de evaluaciones diagnósticas y entrevistas tanto a dichos alumnos ingresantes como a docentes del nivel medio y de primer año de esta facultad. Con estas estrategias arribamos a la conclusión de que estos alumnos no las habían construido en la escuela media ni el curso de ingreso, siendo las mismas necesarias para un buen desempeño en las materias del primer año. Se continuó con un segundo Proyecto: "Las competencias matemáticas y su desarrollo curricular en los primeros años en carreras de ingeniería. El caso de la Facultad Regional Resistencia" (2014-2016), donde se investigó sobre las estrategias de enseñanza y aprendizaje activas que favorezcan la construcción de competencias matemáticas. Posteriormente, el grupo decidió implementar Aprendizaje Basado en Problemas (ABP) para desarrollar algunos temas de Álgebra y Geometría Analítica (A y GA), de primer año de la carrera Ingeniería Electromecánica (IEM). Finalizado el proyecto se continuó con la aplicación de esta estrategia en dicha asignatura. En esta oportunidad, se presenta lo desarrollado hasta ahora y se analizan los resultados obtenidos.

Palabras clave: aprendizaje activo - aprendizaje basado en problemas - competencias matemáticas - ingeniería.

\footnotetext{
${ }^{1}$ Profesora Titular concursada de Algebra y Geometría Analítica de las carreras de Ingeniería de la Facultad Regional Resistencia (FRRe), Universidad Tecnológica Nacional (UTN). Especialista en Docencia Universitaria por la UTN. Investigadora categoría D de la UTN y V del Ministerio. Integrante del Grupo de Investigación Educativa sobre Ingeniería (GIESIN) de la FRRe. Resistencia, Chaco Argentina. Correo electrónico: cgdelvalle2013@gmail.com

${ }^{2}$ Profesora en Matemática y Cosmografía, Universidad Nacional del Nordeste. Especialista en Docencia Universitaria por la UTN. Profesora titular concursada con dedicación exclusiva a cargo de la cátedra Matemática Discreta, Facultad Regional Resistencia (FRRe), Universidad Tecnológica Nacional. Investigadora categoría D de la UTN y V del Ministerio. Integrante del Grupo GIESIN de la FRRe, UTN. Correo electrónico: nfaguilar13@yahoo.com.ar

${ }^{3}$ Ingeniera en Sistemas de Información, Profesora en Disciplinas Industriales por la Facultad Regional Resistencia (FRRe), Universidad Tecnológica Nacional (UTN). Especialista en Docencia Universitaria. Ayudante de primera concursada en las cátedras Algebra y Geometría Analítica, y Matemática Discreta de FRRe, UTN. Integrante del Grupo GIESIN de la FRRe, UTN. Resistencia, Chaco. Correo electrónico: ana.montenegro910@gmail.com
} 
RIIE (2020), Año 11 (14), 82-93.

DOI: http://dx.doi.org/10.30972/riie.11144639
Resultados de la implementación del Aprendizaje Basado en Problemas en una cátedra de Ciencias Básicas en Ingeniería.

\section{Abstract}

The Engineering Educational Research Team (GIESIN) from Resistencia Regional Faculty (FRRe) of the National Technological University (UTN), Argentina, has conducted research about engineering education since 2010. In its first Project called "The relationship between candidates' real competences and the requiered competences of the admitted candidates in the careers taught at FRRe from UTN" (2010-2013), first year students' mathematical skills were analysed through diagnostic evaluations and interviews to the admitted candidates and educators from both secondary school and FRRe first year courses. We reached the conclusion that neither had those students developed mathematical skills at secondary school nor at the university entry preparation course, being those skills necesary for good performance in first year college subjects. In the second Project called "Mathematical competences and its curriculum development in the first year engineering career. The Resistencia Regional Faculty Case" (2014-2016), active teaching and learning strategies which encourage the development of mathematical skills were researched. Subsequently, the research team applied Problem Based Learning (ABP) techniques to develop some Algebra and Analytic Geometry (A y GA) topics from the first year of the Electromechanical Engineering career (IEM). Upon completion of the project, the application of the strategy was continued on this subject. In this opportunity, the study that has been developed is presented and the results obtained are examined.

Key words: active learning - problem based learning - math skills - engineering.

\section{Marco teórico}

En el marco del Proyecto: "Las competencias matemáticas y su desarrollo curricular en los primeros años en carreras de ingeniería. El caso de la Facultad Regional Resistencia" (2014-2016), se propuso aplicar nuevas estrategias de enseñanza y aprendizaje en una cátedra de primer año (Álgebra y Geometría Analítica) de las ingenierías que se cursan en la Facultad Regional Resistencia (FRRe) de la Universidad Tecnológica Nacional (UTN).

En dicha asignatura siempre se aplicaron estrategias de enseñanza y aprendizaje tradicionales, clases expositivas que daban poco lugar a la participación de los estudiantes.

En el proyecto precedente se realizó un exhaustivo análisis de la deserción y el rendimiento académico de los estudiantes de tres cohortes, 2011, 2012 y 2013, de Álgebra y Geometría Analítica. Se detectó que la deserción producida durante esos años fue del 59\% aproximadamente y que, en los exámenes parciales, el tema Transformaciones Lineales presentó una mayor cantidad de desaprobados, cerca del $72 \%$. Esto indujo al grupo de investigación a reflexionar sobre la necesidad de cambiar las estrategias de enseñanza y aprendizaje, buscando incentivar a los estudiantes enfrentándolos, ya desde primer año, con la resolución de problemas contextualizados a la profesión y así permitirles un mayor protagonismo en la construcción de sus aprendizajes.

Como lo afirma Tobón (2008) "Los estudios tradicionales tienen como uno de sus grandes vacíos la dificultad para lograr la pertinencia de la formación, ya que se 
han tendido a realizar sin considerar de forma exhaustiva los retos del contexto actual y futuro" (p.14).

En la misma línea, Morell (2017) plantea que por mucho tiempo se pensó que educación significaba enseñanza y conocimiento sobre enseñar. Pero, los resultados no fueron los esperados: los estudiantes no aprendían ni desarrollaban las competencias requeridas en sus tareas laborales.

La misma autora aclara que las investigaciones realizadas en este sentido mostraron que los individuos aprenden más y mejor si se "involucran intensamente en su educación" y si "colaboran con otros en la resolución de problemas" (p.18). En cuanto al funcionamiento del cerebro y de la mente, plantean que existen dos factores que favorecen el aprendizaje:

- la actividad práctica: aprenden cuando practican, ya que así las neuronas involucradas se activan repetidamente, y

- la emoción: se motivan e involucran más cuando el proceso de aprendizaje les provoca sentimientos agradables.

Por otro lado, Felder \& Brent (2018) definen el aprendizaje activo como todo trabajo en el aula que involucre a los alumnos en actividades distintas a las habituales de mirar y escuchar a un profesor. Señalan que los estudiantes pueden trabajar individualmente 0 en grupos, ser llamados para responder preguntas, resolver problemas, debatir, reflexionar, intercambiar ideas o preguntar.

Diversos autores sostienen que las metodologías activas del tipo aprendizaje cooperativo y aprendizaje basado en problemas/proyectos son las estrategias de aprendizaje que permitirían desarrollar competencias como capacidad de: aplicar conocimientos de matemáticas, trabajar en equipo, comunicarse eficazmente y reconocer la necesidad de tener la capacidad de querer aprender de por vida, entre otras (Gil, Baños, Montoya, Alias y Montoya, 2007).

Ante este desafío, se plantea el enfoque por competencias. Un modelo centrado en el estudiante, promoviendo el desarrollo de competencias y la demostración de su dominio. Así, se involucra a los alumnos en la construcción activa del conocimiento, trabajando junto con el profesor para lograr un aprendizaje significativo. Un ejemplo de trabajar de esta manera es el Aprendizaje Basado en Problemas (Observatorio de Innovación Educativa del Tecnológico de Monterrey, 2015).

Siguiendo esta línea, el Consejo Federal de Decanos de Ingeniería (CONFEDI) y la Asociación Colombiana de Facultades de Ingeniería (ACOFI), elaboraron un libro en el que se caracteriza el "Aprendizaje Centrado en el Estudiante" (ACE). En el marco de esta obra, Cukierman señala que en este tipo de aprendizaje:

el foco está puesto en lo que el estudiante hace para aprender y el profesor es el guía o "facilitador" ya que, desde su conocimiento y experiencia, tanto de su papel docente como de la disciplina de que se trate, configura las estrategias y acciones necesarias para que sea el alumno el que construya el conocimiento. (2018, p.29) 
En el mismo trabajo se menciona que para implementar en la práctica un ACE es de fundamental importancia que se lleve a cabo un Aprendizaje Activo (AA).

Cukierman considera que fue un académico inglés el primero en definir "aprendizaje activo", quien lo describió como "la reflexión sobre la experiencia" y afirmó que "el aprendizaje se alcanza enfocándose en los problemas en un contexto social" (p.32). Además, señala que posteriormente fue definido como "actividades instruccionales que involucran a los estudiantes en hacer cosas y pensar acerca de lo que están haciendo". Por lo que considera que el AA es todo lo que los estudiantes realicen que no sea simplemente estar sentados atendiendo una clase expositiva.

La noción de "competencia" y de "diseño curricular basado en competencias" o "educación basada en competencias" surge en el campo de la formación profesional, en la búsqueda de un ajuste entre la formación obtenida y acreditada y el puesto de trabajo, y en vinculación con los cambios acaecidos en el sistema productivo. El concepto entra así al sistema educativo desde la formación profesional (Mastache, 2007).

Las metodologías de enseñanza y aprendizaje que favorecen la construcción de competencias son las llamadas "activas". En este contexto CONFEDI ha elaborado una "Propuesta de estándares de segunda generación para la acreditación de carreras de ingeniería en la República Argentina" que denominó Libro Rojo (Consejo Federal de Decanos de Ingeniería, 2018). Sus objetivos fueron, entre otros: actualizar y consolidar el actual modelo de formación de ingenieros, consolidar un modelo de aprendizaje centrado en el estudiante, definir un modelo comparable internacionalmente y definir un enfoque basado en competencias y descriptores de conocimiento. Asimismo, se aclara que:

Los graduados de carreras de ingeniería deben tener una adecuada formación general, que les permita adquirir los nuevos conocimientos y herramientas derivados del avance de la ciencia y tecnología. Además, deberán completar y actualizar permanentemente su formación a lo largo de la vida laboral, en el marco informal o en el formal a través del postgrado. (Libro Rojo de CONFEDI, 2018, p.2)

Por lo expuesto, es válido considerar que una innovación pedagógica para construir y desarrollar competencias en una materia de primer año de ingeniería es aplicar el Aprendizaje Basado en Problemas (ABP). Barrows (1986) define la estrategia de Aprendizaje Basado en Problemas como un método de aprendizaje que se fundamenta en el principio de utilizar problemas como punto de partida para la adquisición e integración de los nuevos conocimientos.

Por su parte, Torp y Sage (1999) definen el ABP como "una experiencia pedagógica (práctica) organizada para investigar y resolver problemas que se presentan enredados en el mundo real". Aclaran que "es un organizador del curriculum y también es una estrategia de enseñanza, dos procesos complementarios". Entre las características principales del ABP mencionan que "compromete activamente a los estudiantes como responsables de una situación problemática" y "crea un ambiente de aprendizaje en el que los docentes alientan a los estudiantes a pensar y los guían en su indagación, con lo cual les permiten alcanzar niveles más profundos de comprensión" (p.37). 
Escribano y Del Valle (2008) afirman que "el ABP garantiza tanto la adquisición de conocimientos como el desarrollo de habilidades y actitudes ante el aprendizaje" (p.21). Esta estrategia activa permite centrar el aprendizaje en el estudiante, e introducir en la enseñanza problemas abiertos y más próximos a su desempeño profesional, favoreciendo la posibilidad de interrelacionar distintas materias 0 disciplinas académicas para intentar solucionar un problema, y permitiendo la integración de conocimientos, desarrollando así la competencia del saber hacer en contexto (Fernández y Duarte, 2013).

Algunas características del ABP son:

- los alumnos son sujetos activos, ya que participan constantemente en la adquisición de su conocimiento.

- el profesor es un facilitador del aprendizaje de sus alumnos.

- los problemas a resolver son diseñados o adaptados para lograr el aprendizaje de ciertos objetivos de conocimiento. contenidos.

- el aprendizaje se centra en el alumno y no en el profesor o sólo en los pequeños.

- estimula el trabajo colaborativo en diferentes disciplinas, se trabaja en grupos

- se puede aplicar a diferentes disciplinas del conocimiento. (Instituto Tecnológico y de Estudios Superiores de Monterrey, s.f.)

Durante la aplicación del ABP, el estudiante analiza el problema y se plantea una serie de interrogantes. Para algunas de estas preguntas tendrá respuestas recurriendo a sus conocimientos previos. Pero habrá otras para las cuales deberá buscar información, iniciándose así el proceso de aprendizaje. Es así como el conocimiento nuevo se va construyendo sobre el escenario del conocimiento previo (Nshaho, 2005 citado por Molina Montoya, 2013).

\section{Metodología}

Las investigaciones realizadas estuvieron encuadradas en la metodología de Investigación - Acción (I-A), cumpliéndose con todas sus fases: planificación, acción, observación y reflexión. Como lo afirman Carr \& Kemmis (1988), dichas fases mantuvieron una interrelación constante conformando una espiral autorreflexiva. Esto se transformó en una forma de autoperfeccionamiento de los profesores (Elliott, 1990).

En el proyecto denominado "Relación entre las competencias reales de los aspirantes y las requeridas a los ingresantes en las carreras que se dictan en la FRRe de la U.T.N." (2010-2013), en primer término, se indagó sobre los diseños curriculares de las escuelas de nivel medio de donde provienen dichos jóvenes. A continuación, se analizó la planificación, guías de estudio e instrumentos de evaluación del Seminario Universitario (SU), el curso de ingreso de aprobación obligatoria en dicha facultad.

Se pudo constatar que lo expresado en los diseños curriculares de las escuelas secundarias de las zonas de donde provienen estos estudiantes, no siempre coincide con lo llevado a la práctica. Conclusión a la que se llegó porque tres investigadoras desarrollaron actividades docentes en el nivel medio, y además se entrevistó a docentes del SU que también dictan clases en escuelas secundarias de la región. 
Con respecto al Seminario Universitario, luego del análisis de los materiales didácticos, los exámenes escritos aplicados y las entrevistas realizadas a los docentes y alumnos que participaron del mismo, se concluyó que existe concordancia entre lo expresado en su planificación y lo llevado a cabo durante el desarrollo de dicho curso introductorio (en el dictado de clases, régimen y criterios privilegiados en las evaluaciones aplicadas).

El siguiente paso fue analizar las competencias para el ingreso a primer año de las carreras de ingeniería, definidas por el Consejo Federal de Decanos de Ingeniería (CONFEDI). Éstas se encuentran definidas en la "Declaración de Valparaíso" sobre competencias requeridas para el ingreso a los estudios universitarios en Argentina (CONFEDI, 2014). Refieren a competencias básicas, transversales y específicas.

A las competencias básicas las definen como "capacidades complejas y generales necesarias para cualquier tipo de actividad intelectual" (p.39). Se mencionan: comprensión lectora, producción de textos y resolución de problemas. Por su parte, las competencias transversales "aluden a capacidades claves para los estudios superiores" (p.39), entre ellas se destacan la autonomía en el aprendizaje y las destrezas cognitivas generales. Por último, las competencias específicas "remiten a un conjunto de capacidades relacionadas entre sí, que permiten desempeños satisfactorios en el estudio de las carreras" (p.39). Algunas son: análisis de una función o un fenómeno físico y/o químico sencillo a partir de su representación gráfica y/o a partir de sus ecuaciones matemáticas; reconocimiento y utilización de conceptos en matemática, física, química y biología, y transferencia del conocimiento científico de física, química, matemática y biología a situaciones problemáticas variadas.

Posteriormente, mediante evaluaciones diagnósticas, entrevistas a profesores del Seminario Universitario (SU) y a docentes del nivel medio, además de la aplicación de encuestas a los aspirantes e ingresantes, se llegó a la conclusión de que los mismos no poseían las competencias necesarias para un buen desenvolvimiento en las materias de primer año (Dalfaro, Demuth, Del Valle y Aguilar, 2013). A continuación, se realizó el seguimiento del desempeño académico de los alumnos cursantes de la asignatura Algebra y Geometría Analítica (A y GA), que como se precisó, ya habían aprobado el SU. Para ello se analizaron distintas fuentes de información: planificaciones de la asignatura, entrevistas a profesores y resultados de los parciales de las distintas comisiones correspondientes a las cohortes 2011, 2012 y 2013.

Teniendo en consideración todo lo indagado, se implementó una nueva estrategia de enseñanza de las matemáticas orientada a la construcción de competencias, el Aprendizaje Basado en Problemas. Al mismo tiempo, se realizó el seguimiento sistemático de la misma a través de encuestas y entrevistas realizadas a docentes y alumnos con su correspondiente evaluación.

\section{La aplicación del Aprendizaje Basado en Problemas (ABP)}

En el proyecto "Las competencias matemáticas y su desarrollo curricular en los primeros años en carreras de ingeniería. El caso de la Facultad Regional Resistencia" (2014-2016), luego de investigar sobre distintas estrategias de enseñanza y aprendizaje activas, se inició la aplicación del Aprendizaje Basado en Problemas (ABP). La innovación se llevó a cabo en el año 2014 en la comisión turno noche de la 
cátedra Álgebra y Geometría Analítica, correspondiente a la carrera Ingeniería Electromecánica.

Para decidir qué contenido trabajar con la metodología del ABP, se realizó un estudio estadístico sobre los resultados de los parciales de todas las comisiones de la asignatura elegida (Álgebra y Geometría Analítica), detectando que "Transformaciones Lineales" era el tema con más bajo rendimiento académico. A raíz de lo indagado, se decidió aplicar dicha metodología para desarrollar este tema en la comisión de primer año del turno noche, con una matrícula de 76 estudiantes.

Los problemas de aplicación se buscaron consultando la bibliografía existente en la cátedra, la Biblioteca de la FRRe y sitios de internet. Luego, tuvieron que ser adaptados, ya que en general los que figuran en los libros no se encuentran contextualizados con el futuro desempeño de un ingeniero electromecánico.

En todos los encuentros la aplicación de la innovación pedagógica se llevó a cabo con la participación de los tres docentes y del ayudante de cátedra; un alumno avanzado de Ingeniería Electromecánica. Las clases fueron teórico-prácticas, diferenciándose así de las habituales, donde la teoría y la práctica se desarrollan en distintos días y horarios con diferentes profesores.

Se llevaron a cabo cuatro sesiones de ABP. En la primera, se presentó la metodología de trabajo al grupo clase, se aclararon los roles y funciones de estudiantes y docentes, los objetivos de aprendizaje que se pretendían alcanzar y los procesos de evaluación formativos y sumativos que se realizarían.

Se indicó a los estudiantes que conformen grupos de trabajo por afinidad, con un máximo de cinco integrantes, definan roles y elijan coordinador y secretario. A continuación, se hizo entrega de los problemas a resolver para que comiencen con la tarea. Los docentes recorrieron los grupos, sugiriendo iniciar la actividad con la lectura del problema y un torbellino de ideas, para luego distribuir la búsqueda de información entre los integrantes.

Para la segunda sesión los grupos ya contaban con el material para realizar la búsqueda, trajeron libros de la biblioteca, apuntes de la cátedra y una netbook. Ya se habían organizado por lo que el trabajo fue más ordenado. Algunos grupos prefirieron pasar a trabajar en otras aulas disponibles, esto se pudo llevar a cabo porque eran tres docentes las que estaban presentes para orientarlos cuando surgían dudas. Los profesores tenían una ficha de seguimiento de los grupos donde asentaban la asistencia, la participación de los integrantes y toda observación que consideraron de importancia.

En la tercera sesión, se continuó con el trabajo grupal y al finalizar la misma, entregaron sus producciones en forma escrita. Finalizadas cada una de las sesiones, se hizo una puesta en común para que cada grupo comente sus avances y dificultades. Dichos trabajos fueron corregidos y devueltos con las observaciones pertinentes antes del último encuentro. En la cuarta sesión, se realizaron exposiciones orales. Las presentaciones la hicieron utilizando PowerPoint, cada integrante participó en la explicación de la resolución del problema. 
Por la gran cantidad de grupos y el escaso tiempo disponible, se decidió que el orden de las exposiciones se realice por sorteo antes del inicio, así los que no lograron presentar su trabajo durante la clase, enviaron sus videos.

Al año siguiente se decidió aplicar ABP para dar el tema Sistemas de Ecuaciones Lineales (SEL). El cambio obedeció a varios motivos. En primer lugar, porque SEL se desarrolla en el primer cuatrimestre y se consideró que aplicar la estrategia de ABP contribuiría a la integración de los estudiantes al iniciar el cursado de la materia mediante el trabajo grupal. Otro factor tenido en cuenta fue que Transformaciones Lineales se dicta en el segundo cuatrimestre y para una correcta resolución de los problemas era necesario aplicar SEL, desarrollado entre las primeras unidades de la asignatura. Sumado a esto, SEL es fundamental para la comprensión de varios contenidos de esta materia y para otras del mismo año, como Física y Química.

\section{Resultados a los que se arribó luego de la aplicación de la innovación pedagógica}

Se puede afirmar que los resultados obtenidos fueron muy satisfactorios, tanto para los docentes como para los estudiantes. Esto se comprobó mediante la aplicación de encuestas a los alumnos y entrevistas a ambos, durante las sucesivas aplicaciones del ABP (2014 a 2018).

En general, las encuestas dieron cuenta de una muy buena aceptación por parte de los estudiantes respecto del Aprendizaje Basado en Problemas. Consideraron que promovía su participación en los grupos de trabajo, la integración con sus pares, estimulaba la discusión de un tema con argumentación, los obligaba a consultar bibliografía y sitios web, entre otras competencias.

Cada vez que se tomaron los exámenes parciales, donde se evaluaba el tema dado con la innovación pedagógica, se coordinaron entrevistas, con dos grupos focales: los que aprobaron y los que no aprobaron dichos exámenes.

Los estudiantes del primer grupo, en general, comentaron que los ayudó a aprender a utilizar libros y sitios de internet para estudiar temas nuevos. Aclararon que, si bien al principio se sintieron un poco asustados y desorientados, con el transcurso del tiempo lo superaron. Lograron relacionarse con sus compañeros, aprendieron a escuchar y respetar las ideas del otro.

Los alumnos del segundo grupo opinaron que los malos resultados obtenidos en el parcial se debieron al poco tiempo que le dedicaron a la práctica, al no estudiar suficientemente la teoría. No todos los involucrados participaron y terminaron abandonando el grupo, solo estudiaron la parte que les tocó investigar y no respetaron los roles y las responsabilidades asignadas.

Por otro lado, los profesores entrevistados, manifestaron que fue un gran desafío asumir el nuevo rol, ya que, en el ABP, ni el contenido ni el docente son el centro. En la enseñanza tradicional el profesor cumple el rol de experto y autoridad formal, mientras que en el ABP se debe transformar en tutor y guía facilitador. 
Sienten que se produce un mayor acercamiento con los estudiantes, puesto que, durante las sesiones de trabajo en el aula, tienen la oportunidad de hablar con cada uno de ellos y conocerlos mejor.

La preparación del material didáctico, la diagramación de las sesiones de ABP y la elaboración de los instrumentos de evaluación, entre otras actividades, les demandan mayor tiempo de dedicación. A pesar de esto último, manifestaron su conformidad con esta innovación didáctica.

Luego del análisis de los resultados obtenidos por los estudiantes en la asignatura $A$ y $G A$, se puede afirmar que los resultados fueron muy satisfactorios, ya que aprobaron el examen parcial el $60 \%$ de los alumnos en el primer año de aplicación del ABP. En el segundo año los resultados continuaron mejorando, aprobando el $75 \%$ de los alumnos.

En los años siguientes los resultados fueron similares. A modo de ejemplo, en el año 2018, se aplicó ABP en la comisión B (turno noche) de Ingeniería Electromecánica, mientras que se tomó como curso testigo la comisión A (turno tarde), donde no se aplicó esta metodología de enseñanza. Se pudo observar que el porcentaje de estudiantes que aprobaron el examen parcial en el curso donde se aplicó la innovación fue del $84 \%$, mientras que en el curso testigo fue del $70 \%$. Además, la cantidad de alumnos que desertaron en este trayecto fue menor en la comisión donde se aplicó ABP.

\section{Conclusiones}

Por seis años consecutivos se ha aplicado el Aprendizaje Basado en Problemas, metodología en la que el estudiante constituye el centro del proceso educativo y los profesores son tutores que guían el aprendizaje de sus alumnos.

En cuanto a los docentes, el nuevo rol que les toca asumir no es fácil, deben estar muy bien preparados para responder a los planteos e inquietudes de los estudiantes. Se debe tener en cuenta que en la actualidad los jóvenes tienen acceso a un gran caudal de información. Es necesario que el profesor sea capaz de orientarlos en la elección de fuentes confiables que les permitan realizar sus trabajos con éxito.

Por otro lado, el docente debe tener en claro la necesidad de dar el tiempo suficiente a los alumnos para que logren construir sus aprendizajes. Es preciso que tenga paciencia y evite, en lo posible, desarrollar y explicar los contenidos necesarios para la resolución del problema. Por esta razón, no es posible desarrollar todos los temas de Álgebra y Geometría Analítica con esta metodología. Pero, tratar algunos temas con ABP, posibilita la integración de los alumnos y puede ser el inicio de la conformación de grupos de estudio, que favorecerían la permanencia de los estudiantes en el primer ciclo, donde se produce la mayor deserción en la Facultad Regional Resistencia.

Con este proyecto de investigación educativa se profundizó en el conocimiento y desarrollo de propuestas de formación concretas en el marco de las competencias matemáticas para carreras de Ingeniería. Se contribuyó al desarrollo de ciertos tipos de aprendizajes vinculados con la vida profesional, trabajo en equipo, consulta a 
distintas fuentes de información, manejo del tiempo, entre otros. Además, se intentó minimizar las consecuencias negativas para el alumno y la institución: deserciones y dificultades en el rendimiento académico.

Como este proyecto estuvo enmarcado en la línea de I-A se compartieron los resultados de la innovación con todos los docentes de Álgebra y Geometría Analítica de la FRRe. Esto contribuyó al perfeccionamiento de los profesores de dicha cátedra.

En cuanto a los estudiantes, en las entrevistas y encuestas realizadas se pudo comprobar que se sentían responsables de sus aprendizajes. Los que obtuvieron notas bajas manifestaron verbalmente que no estudiaron lo suficiente, o que solo se limitaron a averiguar la parte que les tocaba buscar y no se interesaron o no pudieron estudiar el resto. En general, estos alumnos no tenían la práctica de estudiar en grupo ni de consultar diversas fuentes para profundizar una temática, en particular en materias como matemática. No es usual que en las escuelas de nivel medio los profesores pidan textos de esta asignatura.

Por todo lo indagado, se puede afirmar que, con esta forma de aprender los estudiantes se sienten más motivados, le encuentran un sentido a lo que están aprendiendo al ver la aplicación de los temas de la asignatura en problemas relacionados con Física, Química y con su carrera obteniendo mejores resultados. Al trabajar en grupo se están acercando de alguna manera a lo que va a ser su futura vida profesional, ya que como ingenieros deberán formar parte de equipos de trabajo y compartir con distintos tipos de personas.

Por último, no se debe olvidar que estos jóvenes que comienzan a estudiar ingeniería, se van a encontrar cuando egresen con un mundo laboral en constante cambio, que va a requerir una actualización y perfeccionamiento en forma permanente. Para ello, es fundamental poseer competencias como las que se construyen durante el proceso del Aprendizaje Basado en Problemas.

\section{Referencias bibliográficas}

Barrows, H. S. (1986). A taxonomy of problem-based learning methods. Medical Education, 20 (6), 481-486. https://doi.org/10.1111/j.1365-2923.1986.tb01386.x

Carr, W. \& Kemmis, S. (1988). Teoría crítica de la enseñanza: La investigación-acción en la formación del profesorado. Barcelona, España: Martínez Roca.

CONFEDI (2014). Declaración de Valparaíso sobre Competencias genéricas de Egreso del Ingeniero Iberoamericano. Recuperado de https://confedi.org.ar//wpcontent/uploads/2020/04/Cuadernillo-de-Competencias-del-CONFEDI.pdf

Cukierman, U. R. (2018). Aprendizaje centrado en el estudiante: un enfoque imprescindible para la educación en ingeniería. En CONFEDI y ACOFI. Aseguramiento de la calidad y mejora de la educación en Ingeniería: Experiencias en América Latina (pp. 27-39). Bogotá, D. C. Colombia: Opciones Gráficas Ed. Ltda. Recuperado de https://confedi.org.ar//wpcontent/uploads/2018/07/Aseguramiento CONFEDI ACOFI-baja.pdf 
Consejo Federal de Decanos de Ingeniería (2018). Libro Rojo. Propuesta de Estándares de Segunda Generación para la Acreditación de Carreras de Ingeniería en la República Argentina. Recuperado de https://confedi.org.ar/download/documentos confedi/LIBRO-ROJO-DE-CONFEDI-Estandaresde-Segunda-Generacion-para-Ingenieria-2018-VFPublicada.pdf

Dalfaro, N., Demuth, P., Del Valle, C. y Aguilar, N. (2013). Las competencias matemáticas y el ingreso universitario en la UTN - Facultad Regional Resistencia. En XIII Coloquio de Gestión Universitaria en América del Sur: 27 al 29 de noviembre de 2013 (p.104). Bs. As., Argentina: Edutecne.

Gil, C., Baños, R., Montoya, F.G., Alias, A. y Montoya, M.D. (2007). Desarrollo de competencias en Ingeniería a través de metodologías activas. Trabajo presentado en las IV Jornadas Internacionales de Innovación Universitaria, Villaviciosa de Odón Madrid. Recuperado de http://abacus.universidadeuropea.es/bitstream/handle/11268/3228/Gil\%20Mont oya,\%20Consolaci\%C3\%B3n.pdf?sequence=1

Elliott, J. (1990). La investigación-acción en educación. Madrid, España: Morata.

Escribano, A. y Del Valle, A. (2008). El Aprendizaje Basado en Problemas: Una propuesta metodológica en Educación Superior. Madrid, España: Narcea.

Brent, R. \& Felder, R. (2018). Resources for Teaching and Learning STEM. Recuperado el 4 de abril de 2019 de http://educationdesignsinc.com/

Fernández, F.H. y Duarte, J.E. (2013). El aprendizaje basado en problemas como estrategia para el desarrollo de las habilidades específicas en estudiantes de ingeniería. Formación universitaria, 6, (5), 29-38. DOI: 10.4067/S071850062013000500005

Instituto Tecnológico y de Estudios Superiores de Monterrey (s.f.). Las estrategias y técnicas didácticas en el rediseño. El Aprendizaje Basado en Problemas como técnica didáctica. Recuperado de http://sitios.itesm.mx/va/dide/documentos/infdoc/abp.pdf

Mastache, A. (2007). Formar personas competentes. Desarrollo de competencias tecnológicas y psicosociales. Bs. As., Argentina: Novedades Educativas.

Molina Montoya, N. P. (2013). El aprendizaje basado en problemas (ABP) como estrategia didáctica. Revista Academia y Virtualidad, 6(1), 53-61. Recuperado de https://dialnet.unirioja.es/servlet/articulo?codigo $=5558104$

Morell, L. (2017). Pasos esenciales para la innovación de currículos de ingeniería y disciplinas afines. Una guía de referencia para navegar este importante proceso. Instituto InnovaHied. Recuperado de https://innovahied.com/product/pasos-esenciales-para-la-innovacion-decurriculos-de-ingenieria-y-disciplinas-afines-ebook/

Observatorio de Innovación educativa Tecnológico de Monterrey (2015). Educación basada en competencias. Recuperado de http://observatorio.itesm.mx/edutrendsebc 
RIIE (2020), Año 11 (14), 82-93.

DOI: http://dx.doi.org/10.30972/riie.11144639
Resultados de la implementación del Aprendizaje Basado en Problemas en una cátedra de Ciencias Básicas en Ingeniería.

Tobón, S. (2008). La formación basada en competencias en la educación superior: el enfoque complejo. Guadalajara, México: Universidad Autónoma de Guadalajara.

Torp, L. y Sage, S. (1999). El aprendizaje basado en problemas. Desde el jardín de infantes hasta la escuela secundaria. Madrid, España: Amorrortu. 